\title{
LA ESCRITURA DE TEXTOS ARGUMENTATIVOS: UNA ACTIVIDAD MÁS SATISFACTORIA GRACIAS A LA EVALUACIÓN COMPARTIDA
}

The writing of argumentative texts: a more satisfactory activity thanks to the shared evaluation

Escrita de textos argumentativos através da avaliação compartilhada

Experiencia educativa desarrollada dentro del Proyecto de Investigación Escritura Analítica y Diversidad Lingüística: cambios evolutivos y microevolutivos desde la Educación Primaria a la Educación Superior (Programa Estatal de Investigación, Desarrollo e Innovación Orientada a los Retos de la Sociedad, Convocatoria 2015, Modalidad 1: Proyectos De I+D+I, EDU2015-65980-R)

\section{María Dolores Alonso-Cortés Fradejas (1) \\ $\mathbf{M}^{\mathrm{a}}$ Teresa Llamazares Prieto (2) \\ Lourdes Zapico Alonso (3)}

(1) Universidad de León, España. Teléfono: +34 987291125. Correo electrónico: mdalof@unileon.es

(2) Universidad de León, España. Teléfono: +34 987291421. Correo electrónico: mtllap@unileon.es

(3) Universidad de León, España. Teléfono: +34 987291421. Correo electrónico: mzapa@unileon.es

\begin{abstract}
Resumen
Las actividades de enseñanza de la escritura que se desarrollan en los distintos niveles educativos no siempre consiguen el objetivo de formar personas capaces de expresarse adecuadamente por escrito y los estudiantes no perciben la composición de textos como una actividad que les pueda resultar útil ni motivadora. La experiencia educativa que se presenta se ha implementado en Educación Primaria, Educación Secundaria y Educación Superior con el fin de revertir esta situación introduciendo procedimientos de evaluación formativa que ayudan al alumnado a mejorar la calidad de los textos analíticos/argumentativos que son los textos académicos que más frecuentemente deben La escritura de textos argumentativos: una actividad más satisfactoria gracias a la evaluación compartida 350
\end{abstract}


escribir. Los resultados de la evaluación de la experiencia muestran que, en todos los niveles educativos, la escritura les ha resultado una actividad más satisfactoria gracias a la introducción de actividades de evaluación entre pares y compartida.

Palabras clave: Textos argumentativos; escritura; evaluación compartida

\begin{abstract}
The activities of writing that are developed in the different educational levels do not always achieve the objective of forming people able to express themselves adequately and students do not perceive the composition of texts as an activity that can be useful or motivating. The educational experience presented has been implemented in Primary Education, Secondary Education and Higher Education in order to reverse this situation by introducing formative evaluation procedures that help students to improve the quality of the analytical/argumentative texts that are the academic texts that students must write more often. The results of the evaluation of experience show that, at all educational levels, writing has proved to be a more satisfactory activity through the introduction of peer-to-peer and shared assessment activities.
\end{abstract}

Keywords: Argumentative texts; writing; shared assessment

\title{
Resumo
}

Atividades de ensino de escrita que se desenvolvem em diferentes níveis de ensino nem sempre alcançar o objectivo da formação de pessoas que podem adequadamente se expressam por escrito e os alunos não percebem a composição como uma atividade que pode ser útil ou motivá-los. A experiência educacional apresentado foi implementado em Educação Básica, Ensino Secundário e do Ensino Superior para reverter esta situação através da introdução de procedimentos de avaliação formativa que ajudam os alunos a melhorar a qualidade dos textos analíticos/argumentativos que são textos acadêmicos eles devem escrever com mais freqüência. Os resultados da avaliação da experiência mostram que, em todos os níveis de ensino, a escrita tem encontrado a atividade mais satisfatório devido à introdução de atividades de avaliação de pares e compartilhada.

Palavras-chave: Textos argumentativos; escrita; avaliação partilhada 


\section{Introducción}

La escritura es útil no solo para comunicar conocimiento sino también para construirlo (Galbraith, 2009). Escribir ayuda a entender mejor el asunto sobre el cual escribimos. Consecuentemente, aprender a escribir ha sido considerado siempre uno de los aprendizajes fundamentales que los escolares han de realizar y así ha quedado reflejado en las sucesivas leyes educativas.

A pesar de ello, las prácticas de enseñanza de la escritura en los distintos niveles educativos no siempre consiguen el objetivo de formar personas capaces de expresarse adecuadamente por escrito y los estudiantes no perciben la composición de textos como una actividad que les pueda resultar útil ni satisfactoria.

Por ello, la secuencia didáctica que presentamos ha sido diseñada e implementada como parte del desarrollo del proyecto de investigación Escritura Analítica y Diversidad Lingüística: cambios evolutivos y microevolutivos desde la Educación Primaria a la Educación Superior (EDU2015-65980-R) que pretende revertir esta situación proporcionando información relevante sobre los procesos evolutivos involucrados en la producción de textos analíticos/argumentativos y sobre las características de las situaciones didácticas que sirven para enriquecer estos procesos facilitando así el proceso de producción y mejorando la calidad de los textos.

\section{Contextualización}

La escritura es útil no solo para comunicar conocimiento sino también para construirlo (Galbraith, 2009). Escribir ayuda a entender mejor el asunto sobre el cual escribimos. Consecuentemente, aprender a escribir ha sido considerado siempre uno de los aprendizajes fundamentales que los escolares han de realizar y así ha quedado reflejado en las sucesivas leyes educativas.

A pesar de ello, las prácticas de enseñanza de la escritura en los distintos niveles educativos no siempre consiguen el objetivo de formar personas capaces de expresarse adecuadamente por escrito y los estudiantes no perciben la composición de textos como una actividad que les pueda resultar útil ni satisfactoria.

Por ello, la secuencia didáctica que presentamos ha sido diseñada e implementada como parte del desarrollo del proyecto de investigación Escritura Analítica y Diversidad Lingüística: cambios evolutivos y microevolutivos desde la 
Educación Primaria a la Educación Superior (EDU2015-65980-R) que pretende revertir esta situación proporcionando información relevante sobre los procesos evolutivos involucrados en la producción de textos analíticos/argumentativos y sobre las características de las situaciones didácticas que sirven para enriquecer estos procesos facilitando así el proceso de producción y mejorando la calidad de los textos.

\section{Diseño y desarrollo}

La secuencia didáctica fue diseñada, junto con otros miembros del equipo del proyecto, por las tres firmantes de este trabajo que también participaron como docentes.

Para su diseño se tomaron en cuenta investigaciones previas y se hizo especial hincapié en los procedimientos de leer para escribir (Delaney, 2008) y hablar para escribir (Boyé, 2002) y en los procedimientos de evaluación formativa y evaluación compartida (López-Pastor, 2017).

La secuencia se desarrolló a lo largo de 7 sesiones durante las que se realizaron las siguientes tareas:

- Motivación y entrenamiento (Sesión 1; 50 minutos): Se explicó a los alumnos los objetivos de la experiencia, estos respondieron a un cuestionario con el que se evaluaba su perfil lingüístico y se les pidió que redactasen un primer texto en el que debían escribir sus reflexiones sobre el tema de la libertad de vestimenta.

- Pre-test (Sesión 2; 20 minutos): Los alumnos escribieron otro texto donde recogieron sus reflexiones sobre el tema de la libertad de desplazamiento de las personas a países distintos del suyo.

- Lectura compartida, análisis de textos y escritura (Sesión 3; 120 minutos): Por un lado, se realizó una lectura compartida de dos textos que presentaban perspectivas diferentes sobre el mismo tema sobre el que habían escrito en la sesión anterior: uno a favor y el otro en contra. Guiados por las docentes, los alumnos debatieron sobre las características lingüísticas y estructurales de ambos textos y comentaron cuáles eran los aspectos que les habían permitido darse cuenta de que los textos mostraban distintos puntos de vista. Por otro lado, llevaron a cabo un análisis de los textos debiendo señalar los argumentos utilizados por los autores para defender su punto de vista así como los datos, ejemplos, etc. con que los apoyaban (evidencias). Finalmente, tuvieron que redactar un nuevo texto aprovechando lo que en esa sesión habían aprendido. 
- Evaluación entre iguales (Sesión 4; 50 minutos): Se les dijo a los alumnos que iban a evaluar los textos escritos por sus compañeros en la sesión anterior y se les presentó la rúbrica que iban a utilizar para hacerlo y que sería la misma que luego usarían las docentes para valorar sus producciones. Situados en parejas, evaluaron los textos primero de forma individual y luego conjuntamente.

- Evaluación compartida (Sesión 5; 50 minutos): Tras recoger los resultados de las evaluaciones conjuntas en una tabla de doble entrada, las docentes los expusieron al grupo y los alumnos extrajeron conclusiones sobre los indicadores que habían resultado mejor y peor valorados y, por lo tanto, sobre los puntos fuertes y más débiles de sus escritos.

- Aplicación de aprendizajes (Sesión 6; 20 minutos): Se les pidió a los alumnos que aplicaran lo que habían aprendido sobre las características de un buen texto explicativo/argumentativo para redactar un nuevo texto sobre el tema de la libertad de desplazamiento de las personas a países distintos del suyo.

En todos los niveles y grupos los textos se redactaron con ordenador.

\section{Evaluación y conclusiones}

Al finalizar la experiencia se evaluó la satisfacción de los participantes en la experiencia. Los alumnos se mostraron, en general, altamente satisfechos manifestando que habían aprendido mucho y que la tarea que más les había gustado era la de evaluar los textos de los compañeros. Lo que valoraron más negativamente fue el tener que redactar varias veces el texto sin poder volver sobre sus anteriores producciones para modificarlas. Resultó interesante comprobar que hubo un alto grado de coincidencia las valoraciones de los alumnos de los distintos niveles educativos.

Por su parte, las docentes se mostraron asimismo muy satisfechas si bien realizaron varias sugerencias de mejora: entre ellas, introducir un proceso de autoevaluación y enriquecer la retroalimentación con gráficos y los resultados de la evaluación de los textos por ellas.

A la espera de que el análisis de las evaluaciones de los textos producidos antes y después de las sesiones de lectura compartida y evaluación formativa confirme nuestra hipótesis de que los textos finales tendrán mejor calidad, podemos avanzar como una conclusión de nuestro proyecto de investigación que la introducción de procedimientos 
de evaluación formativa y compartida incrementa la satisfacción de los alumnos respecto a la realización de tareas de escritura habitualmente consideradas poco satisfactorias.

\section{Referencias}

Boyé, O. G. (2002). Hablar para escribir. Aula de innovación educativa, (111), 15-17.

Delaney, Y. A. (2008). Investigating the reading-to-write construct. Journal of English for Academic Purposes, 7(3), 140-150.

Galbraith, D. (2009). Writing about what we know: Generating ideas in writing. En A R. Beard, D. Myhill, M. Nystrand, \& J. Riley (eds.), The SAGE handbook of writing development. (pp. 48-65). London: SAGE.

López-Pastor, V.M. (2017). Evaluación formativa y compartida: evaluar para aprender y la implicación del alumnado en los procesos de evaluación y aprendizaje. En V.M. López-Pastor \& Á. Pérez-Pueyo, Evaluación formativa y compartida en educación: experiencias de éxito en todas las etapas educativas (pp. 34-68). León: Universidad de León. 